\title{
Induction of cell cycle arrest by increasing GTP-RhoA levels via Taxol-induced microtubule polymerization in renal cell carcinoma
}

\author{
YU REN ${ }^{1,2}$, XUE WANG $^{1,2}$, ZHONGGUAN LOU $^{1,2}$, SHUAISHUAI HUANG $^{1,2}$, \\ HAIHUI ZHUANG ${ }^{3}$, YUDUO WANG ${ }^{3}$, GUOBIN WENG $^{1,2}$ and PING WANG ${ }^{3}$
}

\author{
${ }^{1}$ Department of Urologic Surgery, Ningbo Urology and Nephrology Hospital; ${ }^{2}$ Laboratory of Kidney Carcinoma, \\ Urology and Nephrology Institute of Ningbo University, Ningbo, Zhejiang $315000 ;{ }^{3}$ Center for Translational Medicine, \\ Ningbo University School of Medicine, Ningbo, Zhejiang 315211, P.R. China
}

Received January 19, 2016; Accepted January 20, 2017

DOI: $10.3892 / \mathrm{mmr} .2017 .6543$

\begin{abstract}
Renal cell carcinoma (RCC) is the most common neoplasm of the kidney in adults, accounting for $\sim 3 \%$ of adult malignancies. Understanding the underlying mechanism of RCC tumorigenesis is necessary to improve patient survival. The present study revealed that Taxol-induced microtubule (MT) polymerization causes cell cycle arrest and an increase in guanosine triphosphate-Ras homology gene family, member A (GTP-RhoA) protein expression. Disruption of Taxol-induced MT polymerization reversed GTP-RhoA expression and cell cycle arrest. The localization and redistribution of MTs and RhoA were consistent in cells with MT bundles and those without. Decreased GTP-RhoA had no marked effect on Taxol-induced MT bundling, however, it reduced the proportion of cells in G2/M phase. Taken together, Taxol-induced MT polymerization regulated the protein expression levels of GTP-RhoA and cell cycle arrest. However, the alteration in GTP-RhoA expression did not influence MT arrangement, suggesting that GTP-RhoA serves a pivotal role in Taxol-induced MT polymerization and cell cycle arrest in RCC.
\end{abstract}

\section{Introduction}

Renal cell carcinoma (RCC) is the most common neoplasm of the kidney in adults, accounting for $\sim 3 \%$ of adult malignancies (1). Surgical resection remains the only definitive treatment for RCC (2). However, following surgery, 20-40\% of

Correspondence to: Professor Guobin Weng, Department of Urologic Surgery, Ningbo Urology and Nephrology Hospital, 1 Qianhe Road, Ningbo, Zhejiang 315000, P.R. China

E-mail: nbsys098@126.com

Dr Ping Wang, Center for Translational Medicine, Ningbo University School of Medicine, 818 Fenghua Road, Ningbo, Zhejiang 315211, P.R. China

E-mail:pinoav@hotmail.com

Key words: guanosine triphosphate-ras homolog gene family, member A, microtubule, cell cycle, Taxol, renal cell carcinoma patients will eventually relapse due to developing resistance to other treatment regimens, for example chemotherapy and radiotherapy (3). Therefore, there is an ongoing requirement for novel therapeutic strategies and a greater understanding of the underlying mechanisms involved in the development of RCC.

Ras homology gene family, member A (RhoA) is a member of the Ras-superfamily of small guanosine triphosphatases (4) and is a pivotal control point by which cells sense alterations in extracellular matrix and cytoskeletal organization. RhoA translates these signals to downstream effectors to mediate cell proliferation and motility $(5,6)$. Active guanosine triphosphate (GTP)-bound RhoA causes the formation of stress fibers by activation of downstream Rho-associated kinases (7), which enhance the formation of actin/myosin microfilaments (MF) to facilitate cell motility (8). A previous report suggested that the localization of RhoA and its effector, mammalian diaphanous-related formin-1, control microtubule (MT) dynamics, the actin network and adhesion site formation in migrating cells (9). Our previous studies revealed that the formation of an MT ring structure supported apoptotic cell morphology (10-12). However, little is known about the role of RhoA in MT polymerization.

The present study used Taxol, a common MT stabilizer that promotes tubulin polymerization (13), to investigate the association between RhoA and MT arrangement.

\section{Materials and methods}

Cell culture. The RCC cell line OS-RC-2, was obtained from the Cell Bank of the Chinese Academy of Sciences (Shanghai, China). Cells were cultured in RPMI 1640 medium, supplemented with $10 \%$ heat-inactivated fetal bovine serum, $100 \mathrm{U} / \mathrm{ml}$ penicillin and $100 \mu \mathrm{g} / \mathrm{ml}$ streptomycin (all from HyClone; GE Healthcare Life Sciences, Logan, UT, USA), in a humidified incubator at $37^{\circ} \mathrm{C}$ and $5 \% \mathrm{CO}_{2}$ (14). Following culture for $24 \mathrm{~h}$, OS-RC-2 cells were treated with $1 \mu \mathrm{M}$ Taxol (Shanghai Hualian Pharmaceutical Co., Ltd., Shanghai, China) for $0,12,24,36$ and $48 \mathrm{~h}$. Alternatively, following culture for $24 \mathrm{~h}$, the cells were pretreated with $10 \mu \mathrm{M}$ MT depolymerizing agent Colchicine (Col; cat. no. C8190; Beijing Solarbio Science \& Technology Co., Ltd., Beijing, China) or $2 \mu \mathrm{g} / \mathrm{ml}$ 
RhoA inhibitor C3 transferase (cat. no. CT03-A; Cytoskeleton, Inc., Denver, CO, USA) for $1 \mathrm{~h}$, which was followed by treatment with $1 \mu \mathrm{M}$ Taxol for $24 \mathrm{~h} 37^{\circ} \mathrm{C}$ and $5 \% \mathrm{CO}_{2}$. Untreated cells served as the control.

Immunofluorescent staining of MF, MT and RhoA. Cells were stained for MF, MT and RhoA as previously described $(15,16)$. Coverslips were washed twice with phosphate-buffered saline (PBS) and examined under a confocal scanning microscope (Bio-Rad Laboratories, Inc., Hercules, CA, USA).

Transfection experiments. The pCI-neo empty vector were provided by Professor Ningsheng Liu, Nanjing Medical University (Nanjing, China). The control vector was produced by inserting the RhoA coding DNA sequence into the EcoRI-Sal I site of pCI-neo vector. The dominant-negative mutants vector RhoAT19N was constructed using Asp instead of Thr188 (mutations $849 \mathrm{~A} \rightarrow \mathrm{T}$ and $850 \mathrm{~T} \rightarrow \mathrm{G}$ ) using the site-directed mutagenesis kit (cat. no. 200517; Agilent Technologies, Inc., Santa Clara, CA, USA). Cells were plated in $25-\mathrm{cm}^{2}$ culture flasks at a density of $5 \times 10^{5}$ cells/flask. Following culture for $24 \mathrm{~h}$, cells were transiently transfected with the control vector and RhoAT19N vector. Transfection was performed using Lipofectamine ${ }^{\circledR} 2000$ (Invitrogen; Thermo Fisher Scientific, Inc., Waltham, MA, USA) in accordance with the manufacturer's protocol. The concentration of the DNA plasmid used was determined by the size of culture dishes, also according to the manufacturer's instructions. Cells were analyzed at $40 \mathrm{~h}$ following transfection (17).

Cell cycle measurements. For flow cytometric analysis of the cell cycle, $1 \times 10^{6}$ cells were harvested by centrifugation (room temperature, $184 \mathrm{x} \mathrm{g}, 5 \mathrm{~min}$ ), washed with PBS and fixed with ice-cold $70 \%$ ethanol overnight. Fixed cells were treated with $25 \mu \mathrm{g} / \mathrm{ml}$ RNase A at $37^{\circ} \mathrm{C}$ for $30 \mathrm{~min}$ and stained with $50 \mu \mathrm{g} / \mathrm{ml}$ propidium iodide (Sigma Aldrich; Merck Millipore, Darmstadt, Germany) for $30 \mathrm{~min}$ in the dark. The fluorescence intensity of individual cells was measured using a flow cytometer (Beckman Coulter, Inc., Miami, FL, USA). At least 10,000 cells were counted using CXP Cytometer version 2.2 software (Beckman Coulter, Inc.) (18).

RhoA protein expression. The expression of RhoA protein was measured by flow cytometry as previously described (6). Briefly, $1 \times 10^{6}$ cells were fixed with $4 \%$ (w/v) paraformaldehyde for $30 \mathrm{~min}$ at $4^{\circ} \mathrm{C}$, and permeabilized using $0.1 \%$ Triton $\mathrm{X}-100$. Nonspecific antibody binding was blocked by incubation in $0.5 \%$ bovine serum albumin (BSA; Beijing Solarbio Science $\&$ Technology Co., Ltd.) for $30 \mathrm{~min}$ at room temperature. Cells were subsequently incubated with primary RhoA antibodies (dilution, 1:500; cat. no. sc-418; Santa Cruz Biotechnology, Inc.) in $0.01 \mathrm{M} \mathrm{PBS}$ at $4^{\circ} \mathrm{C}$ overnight. Following this, the cells were incubated with a FITC-conjugated anti-mouse secondary antibody (dilution, 1:50; cat. no. BA1101; Wuhan Boster Biological Technology, Ltd., Wuhan, China) for $1 \mathrm{~h}$ at room temperature in the dark. The expression of RhoA was then measured by flow cytometry (BD Biosciences, San Jose, CA, USA).

Western blot analysis. The OS-RC-2 cells were lyzed in ice-cold radioimmunoprecipitation buffer (cat. no. R0010; Beijing
Solarbio Science \& Technology Co., Ltd.) containing PMSF protease inhibitor (cat. no. Amresco0754; Beijing Solarbio Science \& Technology Co., Ltd.) and phosphatase inhibitors (cat. no. C500017; Sangon Biotech Co., Ltd., Shanghai, China). Equal quantities of protein $(40 \mu \mathrm{g})$ were separated by $12 \%$ SDS-PAGE, prior to transferring proteins to polyvinylidene difluoride membranes (0.4 $\mu \mathrm{m}$; EMD Millipore, Billerica, MA, USA). The membranes were blocked in 5\% BSA in TBST (0.1\% Tween-20) with $0.2 \%$ sodium azide and incubated with primary antibodies: RhoA (dilution, 1:500; cat. no. sc-418; Santa Cruz Biotechnology, Inc.), GTP-RhoA (dilution, 1:500; cat. no. 80601; Neweast Biosciences, King of Prussia, PA, USA) or GAPDH (dilution, 1:1,000; cat. no. 2118S; Cell Signaling Technology, Danvers, MA, USA) overnight at $4^{\circ} \mathrm{C}$. Following washing, membranes were incubated with a horseradish peroxidase-conjugated goat anti-mouse/rabbit IgG secondary antibody (dilution, 1:5000; cat. no. BA1050/BA1054; Wuhan Boster Biological Technology, Ltd.) at room temperature for $2 \mathrm{~h}$, and chemiluminescence was performed using an Enhanced Chemiluminescence Detection kit (Shanghai Biyuntian Biological Co., Ltd., Shanghai, China), according to the manufacturer's protocol, with Tanon MP version 4.1.2 software (Tanon Science and Technology Co., Ltd., Shanghai, China) (19).

Statistical analysis. All experiments were repeated three times. Data are expressed as the mean \pm standard deviation. One-way analysis of the variance and Fisher's least significant difference post hoc tests were performed to evaluate the differences among groups by SPSS v13.0 software (SPSS, Inc., Chicago, USA). $\mathrm{P}<0.05$ was considered to indicate a statistically significant difference.

\section{Results}

Taxol induces MT polymerization, cell cycle arrest and $G T P-R h o A$ protein expression in $O S-R C-2$ cells. Taxol is an effective chemotherapeutic used to treat cancer patients; it binds to and stabilizes MT, causing abnormal MT aggregation (20). Immunofluorescent staining revealed that in the absence of Taxol, tubulin was widely distributed throughout the cytoplasm and accumulated in the perinuclear regions of cells. However, in cells treated with Taxol, a distinct rearrangement of MT was observed, including the formation of rigid MT bundles in the cytoplasm and the area surrounding the nucleus (Fig. 1A).

To determine the effect of Taxol-induced MT polymerization on the expression of GTP-RhoA and the cell cycle, western blotting and flow cytometric analysis were performed. Expression of GTP-RhoA was significantly upregulated following Taxol treatment in a time-dependent manner (Figs. 1B and C). Additionally, Taxol treatment caused an increase in GTP-RhoA protein expression in a dose-dependent manner (data not shown). The proportion of cells in $\mathrm{G} 2 / \mathrm{M}$ phase increased >4-fold in cells treated with Taxol for $12 \mathrm{~h}$, compared with the control (Fig. 1D). Therefore, these results suggested that Taxol-induced MT polymerization is accompanied by an increase in GTP-RhoA protein expression levels and cell cycle arrest.

Taxol treatment causes concurrent redistribution of MT and GTP-RhoA. To investigate the potential association between 
A

MFs

MTs

Nuclei

Merge
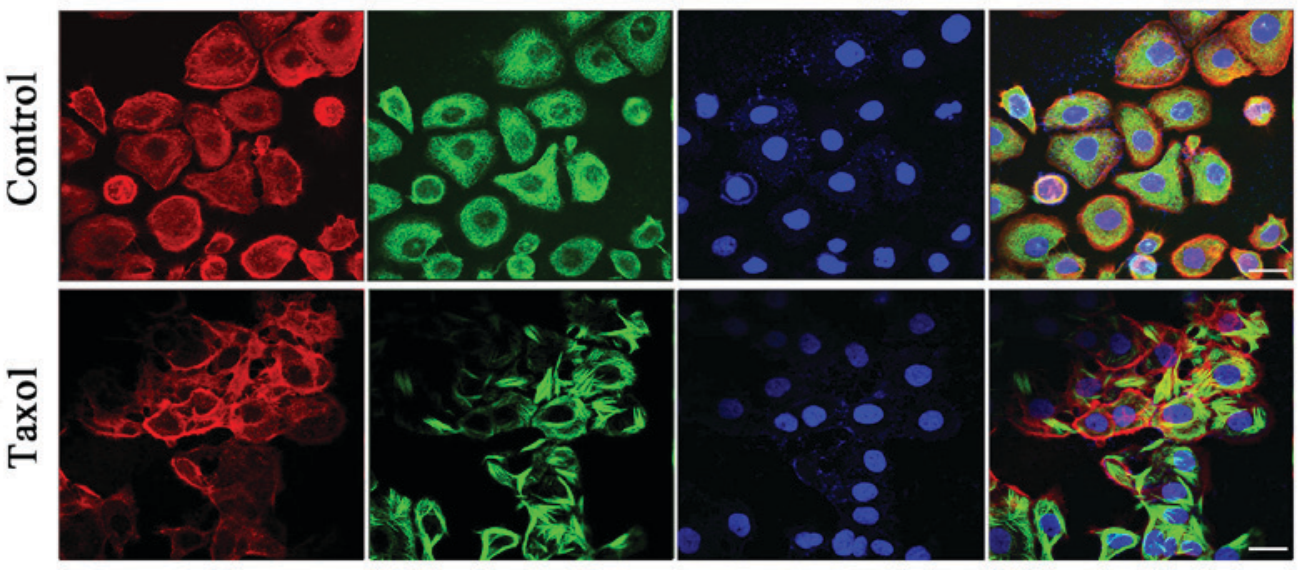

B
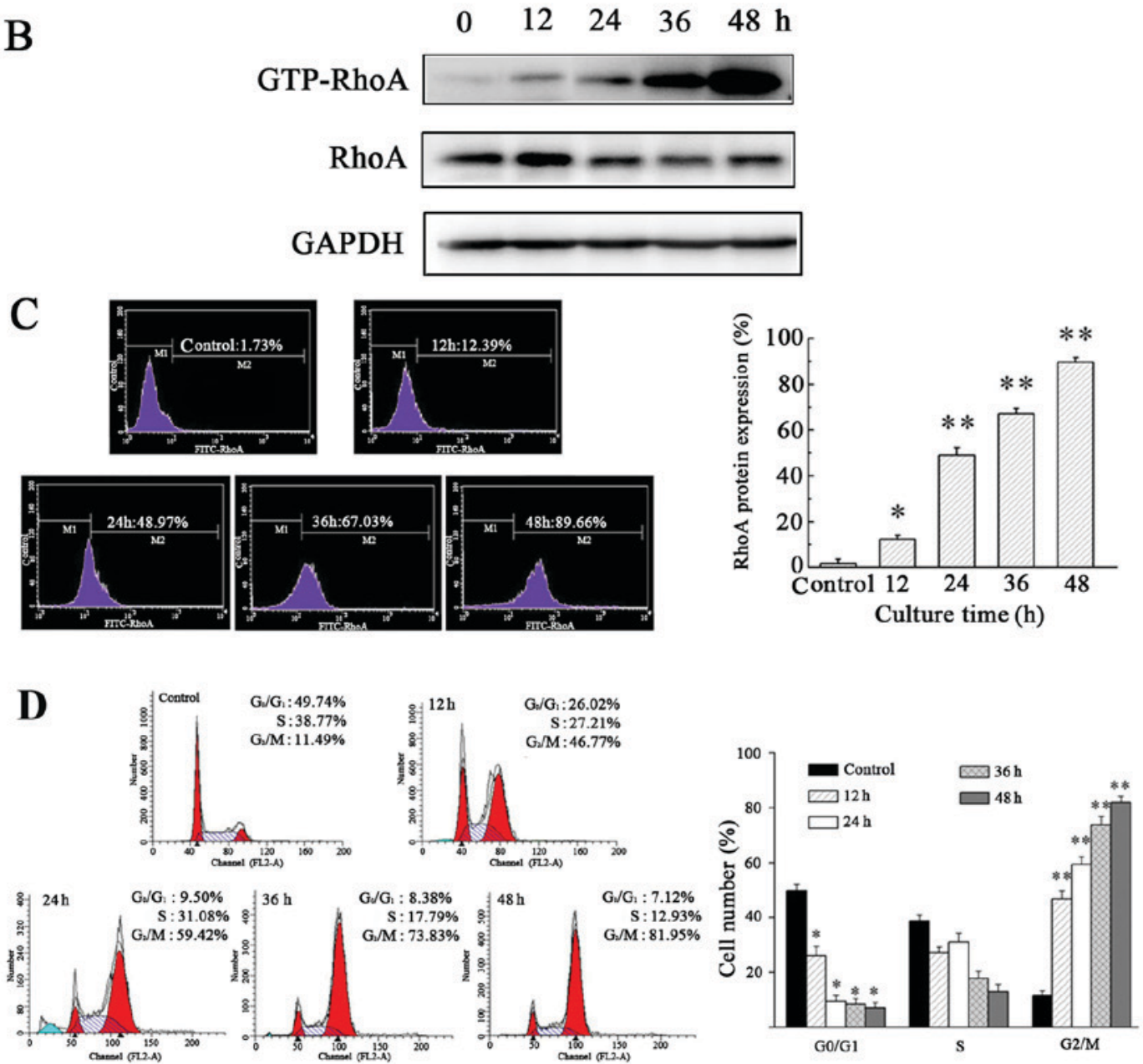

Figure 1. Taxol-induced MT polymerization increases GTP-RhoA protein expression and cell cycle arrest. MT polymerization was elevated following Taxol treatment, as determined by immunofluorescent staining and confocal microscopy. (A) Confocal images of MFs stained with rhodamine-phalloidin (red), MTs stained with Alexa 488-labeled $\beta$-tubulin antibody (green) and nuclei stained with DAPI (blue) are presented. Scale bar=10 $\mu \mathrm{m}$. (B) Western blot analysis and (C) Flow cytometry assessed GTP-RhoA protein expression levels following Taxol treatment. Expression of GTP-RhoA was significantly upregulated in a time-dependent manner. (D) Taxol treatment caused a significant increase in the proportion of cells in G2/M phase, as determined by flow cytometry. Data are presented as the mean \pm standard deviation of three independent experiments. ${ }^{*} \mathrm{P}<0.05$ and ${ }^{* * *} \mathrm{P}<0.01$ vs. control. MT, microtubule; GTP, guanosine triphosphate; RhoA, Ras homology gene family, member A; MF, actin/myosin microfilaments.

GTP-RhoA and MT arrangement, their cellular localization was determined via immunofluorescent staining. The results revealed a consistent co-localization of GTP-RhoA and MT structure (yellow colorization). GTP-RhoA and MT were widely distributed in control cells. The GTP-RhoA protein was located adjacent to the MT bundles in cells treated with Taxol. Following treatment with the MT depolymerizing agent $(\mathrm{Col})$, the MT bundles were partially disrupted and the GTP-RhoA 

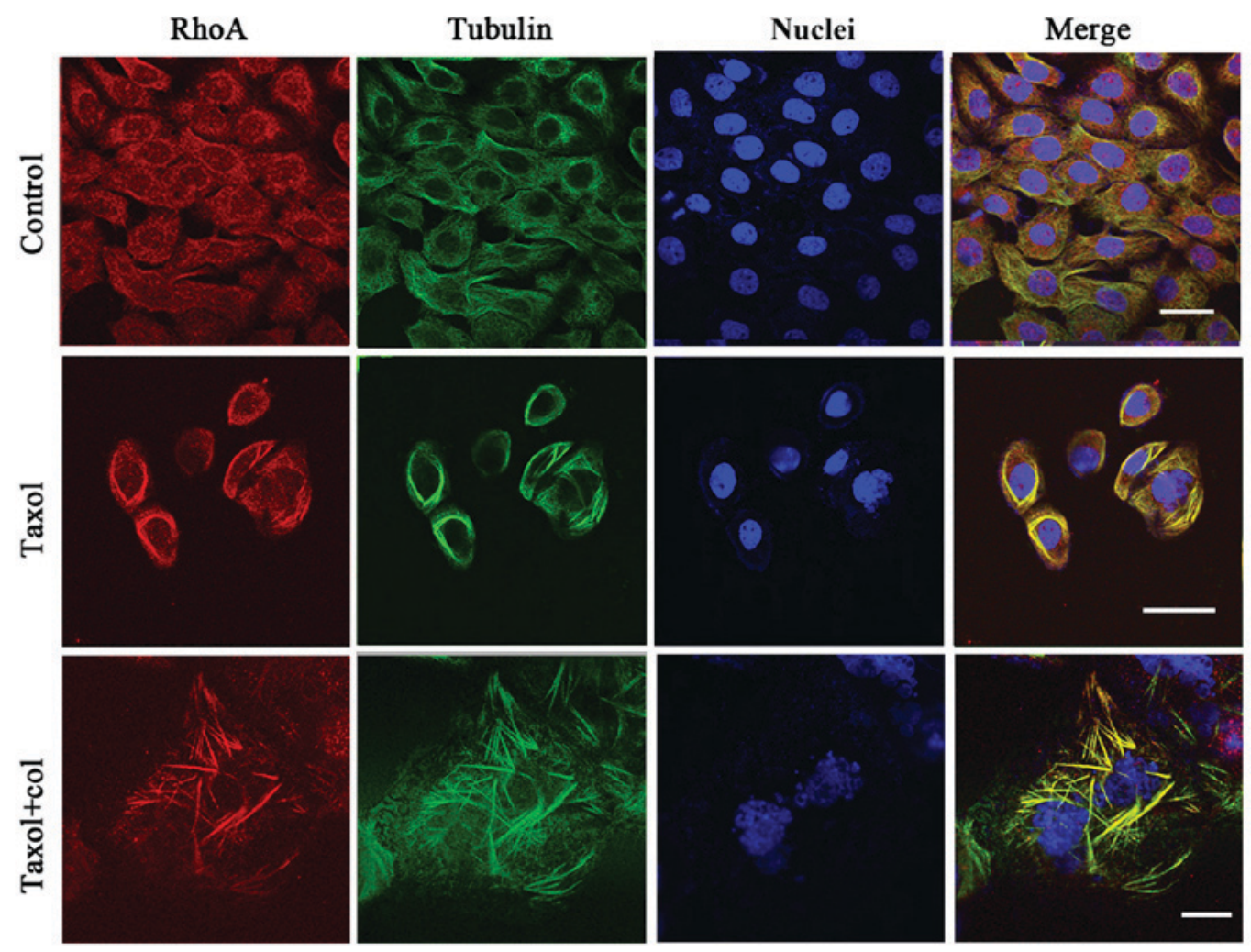

Figure 2. Taxol treatment of cells induces MT and GTP-RhoA co-localization, as determined by immunofluorescent staining. Confocal images demonstrate cell staining of RhoA (red), MTs (green) and nuclei (blue). Scale bar=10 $\mu \mathrm{m}$. MT, microtubule; GTP, guanosine triphosphate; RhoA, Ras homology gene family, member A.

distribution dispersed. The results demonstrated a concurrent redistribution of MT arrangement and GTP-RhoA in Taxol treated cells (Fig. 2).

Disruption of Taxol-induced MT polymerization decreases GTP-RhoA protein expression and reverses cell cycle arrest. Next, the association between MT polymerization and GTP-RhoA protein expression was investigated. Combined treatment with Col and Taxol disrupted Taxol-induced MT bundling, however, little effect on MF arrangement was detected (Fig. 3A). The combination of $\mathrm{Col}$ and Taxol abolished Taxol-induced GTP-RhoA protein expression levels to the levels of the untreated control, as determined by western blotting (Fig. 3B) and flow cytometric analysis (Fig. 3C), and the Taxol-induced increase in the proportion of cells in G2/M phase was significantly reduced (Fig. 3D). These results suggested that MT arrangement regulated the protein expression levels of GTP-RhoA and cell cycle arrest.

Inhibition of GTP-RhoA has no effect on Taxol-induced MT bundling but inhibits the Taxol-induced increase in G2/M cell number. To investigate the effect of GTP-RhoA on cytoskeleton arrangement and cell cycle, cells were pretreated with a RhoA inhibitor (C3 transferase) for $1 \mathrm{~h}$ or transfected with a RhoAT19N mutant to decrease GTP-RhoA expression (Fig. 4A). The inhibition of GTP-RhoA expression partially reduced the proportion of cells in G2/M compared with Taxol treatment alone (Fig. 4B and C). However, no marked effect on MT arrangement was observed in cells treated with the combination of C3 transferase or RhoAT19N mutant and Taxol (Fig. 4D). Thus, these findings confirmed that the cell cycle was affected by alterations in RhoA expression; however, GTP-RhoA expression did not influence MT arrangement.

\section{Discussion}

Taxol is a member of the taxane class of antineoplastic microtubule damaging agents and is widely used to treat a variety of human malignancies, including breast, ovarian, lung, prostate and bladder cancers. Taxol may stabilize microtubules and subsequently cause cell death by arresting the cell cycle at $\mathrm{G} 2 / \mathrm{M}(21,22)$. The present study demonstrated that Taxol-induced MT polymerization is accompanied by an increase in GTP-RhoA protein expression levels and cell cycle arrest in RCC cells. A previous report revealed that disruption of MT arrangement causes enhanced GTP-RhoA activity via release of an MT-associated Rho activator, guanine nucleotide exchange factor (GEF) -H1 (GEF-H1) (23). Downstream of RhoA, Rho-associated protein kinase (ROCK) signaling serves a pivotal role in the rescue of the mutant huntingtin-expressing cells from cell death caused by MT depolymerizing agents (23). Furthermore, a recent report has discussed a similar feedback loop between the Rho/ROCK signaling pathway and MT dynamics in an acute Tlymphoblastic leukemia cell line $(24,25)$. The mechanism underlying MT rearrangement-associated alteration of GTP-RhoA expression remains to be fully understood. Therefore, the present study investigated the regulatory association between the MT cytoskeleton and cell cycle progression, in particular, the involvement of GTP-RhoA. 
A

MFs

MTs

Nuclei

Merge
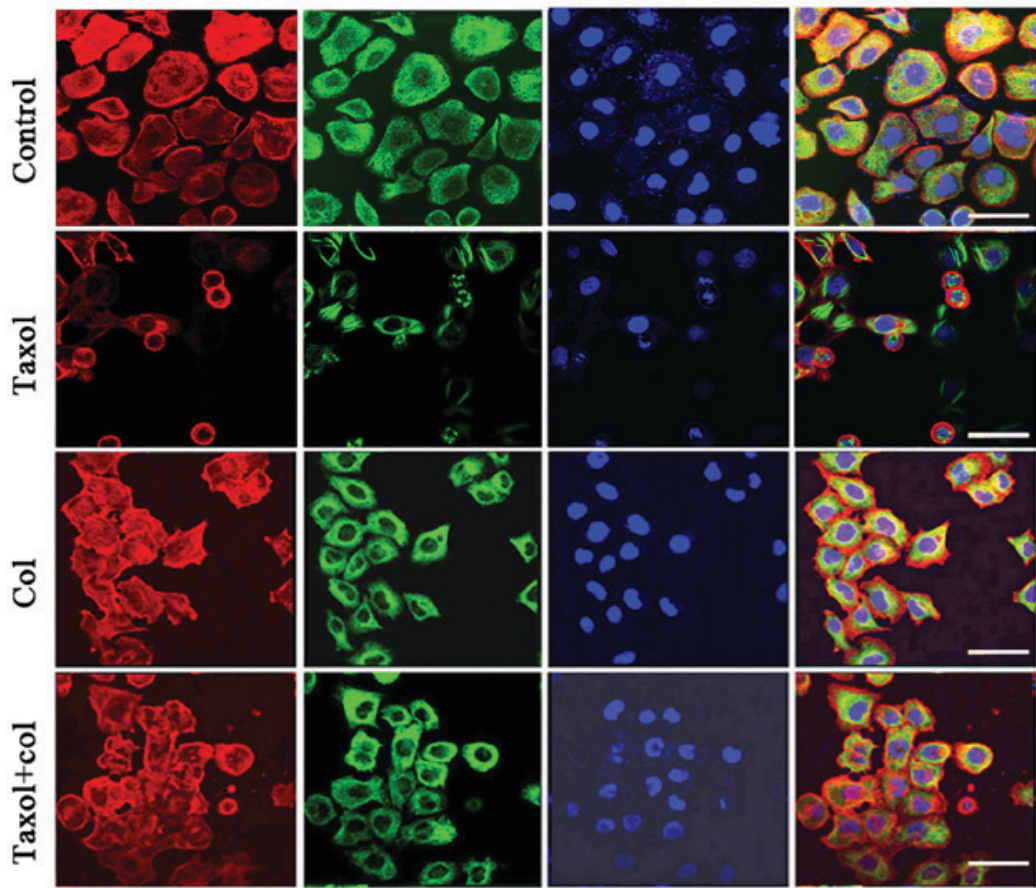

B

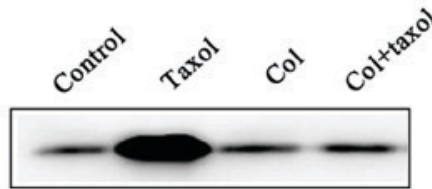

GTP-RhoA

RhoA

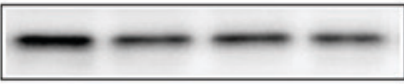

\section{GAPDH}

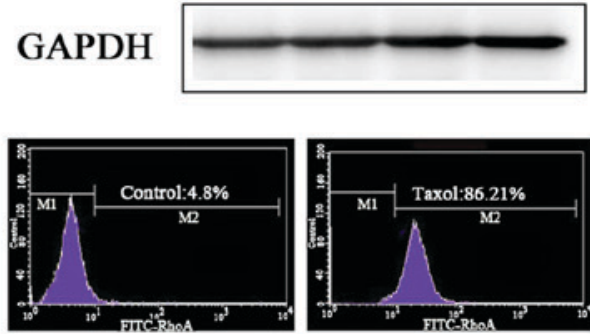

C
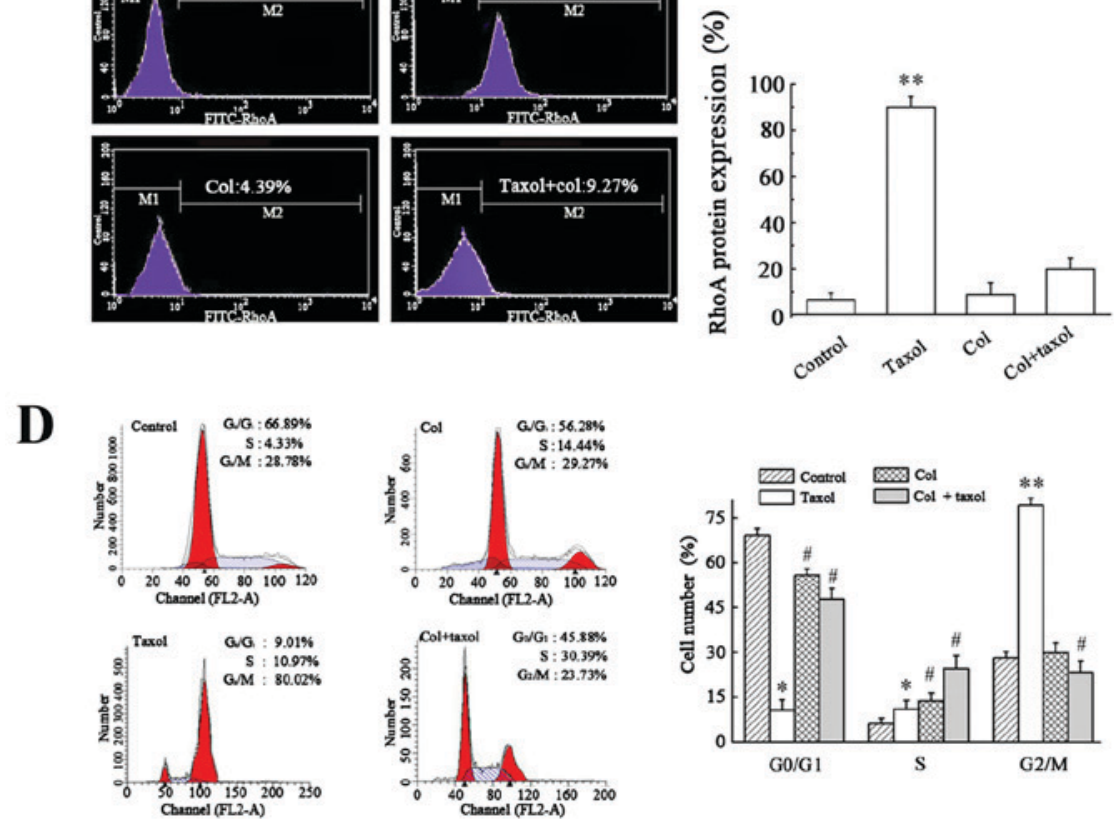

Figure 3. Disruption of Taxol-induced MT polymerization decreases GTP-RhoA protein expression levels and reverses cell cycle arrest. Cells pre-treated with the MT depolymerizing agent, Col, (A) exhibited reduced MT bundling and (B) GTP-RhoA protein expression levels, which was determined by western blotting and (C) flow cytometry. (D) Taxol-induced cell cycle arrest was reversed following col treatment. Data are presented as the mean \pm standard deviation of three independent experiments. ${ }^{*} \mathrm{P}<0.05$ and ${ }^{* *} \mathrm{P}<0.01$ vs. control; ${ }^{*} \mathrm{P}<0.05$ vs. Taxol. Scale bar=10 $\mu \mathrm{m}$. MT, microtubule; GTP, guanosine triphosphate; RhoA, Ras homology gene family, member A; Col, colchicine; MF, actin/myosin microfilaments. 
A

$\mathrm{C}$

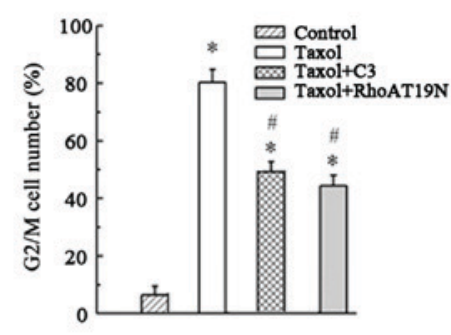

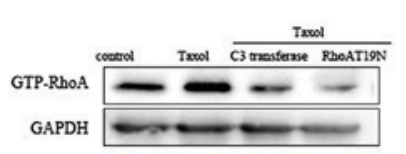

B

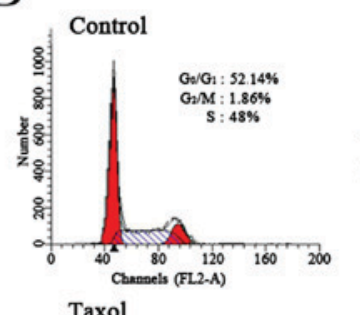

Taxol

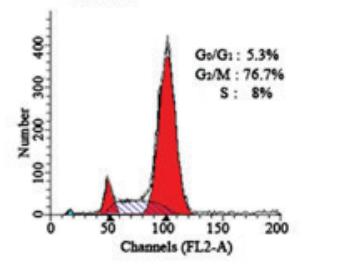

Chanoels (FL2.A)

D

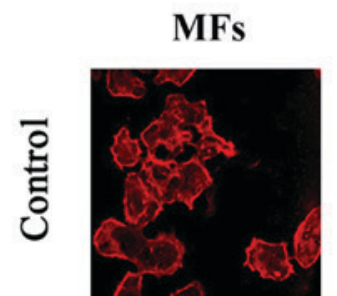

MTs
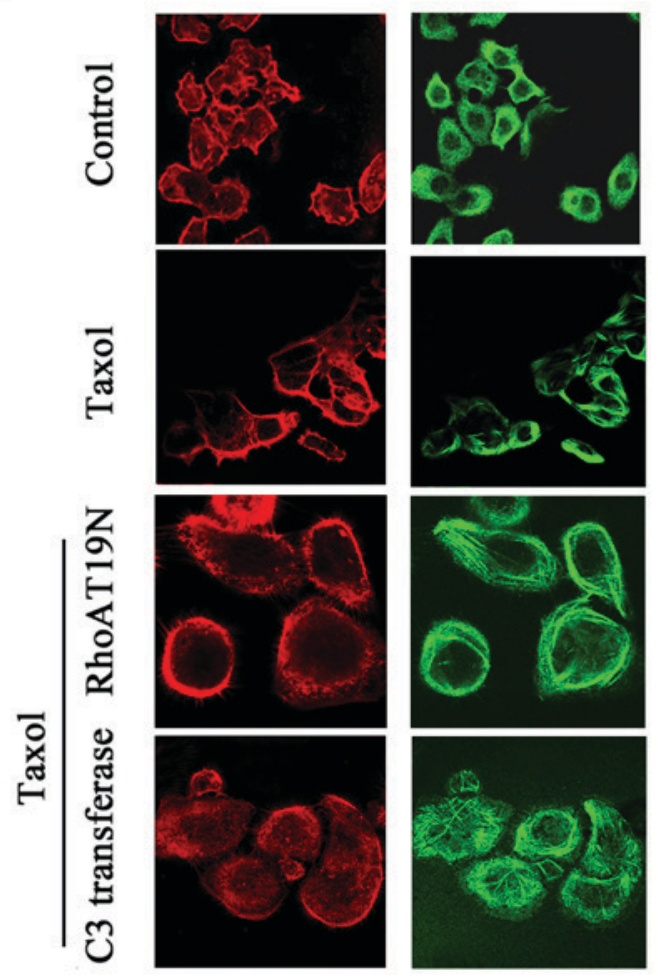

Nuclei
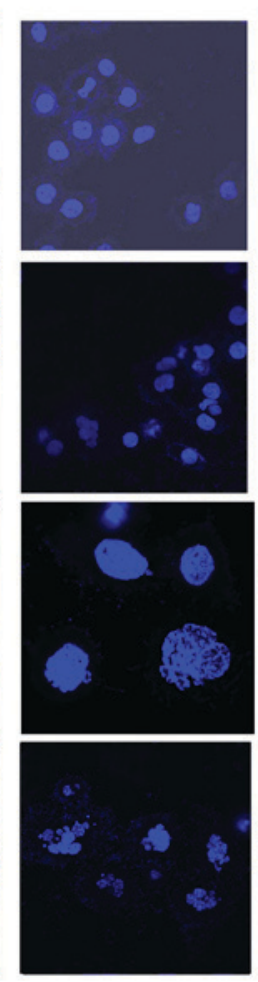

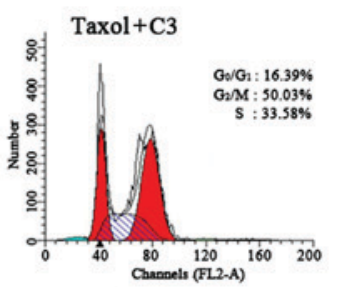

Taxol+RhoAT19N

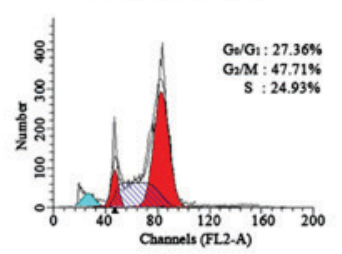

Merge
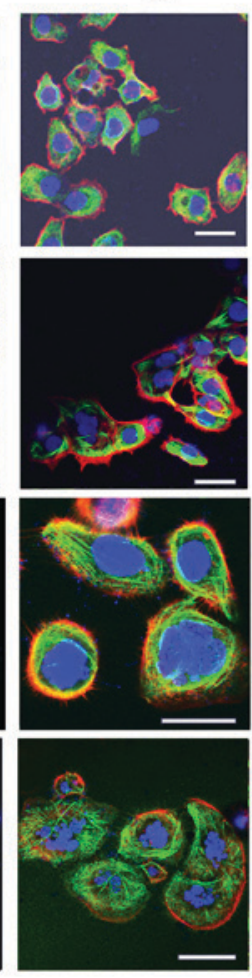

Figure 4. GTP-RhoA inhibition has no effect on Taxol-induced MT bundling, but partially inhibits the Taxol-induced increase in G2/M cell number. (A) Western blotting demonstrated that transfection with the RhoAT19N mutant or using RhoA inhibitor (C3 transferase) significantly decreased GTP-RhoA expression. (B and C) The combination of Taxol with RhoAT19N plasmid or C3 transferase decreased the Taxol-induced increase in the proportion of cells in the G2/M phase however, (D) it had no marked effects on Taxol-induced MT bundling. Data are presented as the mean \pm standard deviation of three independent experiments. "P<0.05 vs. control; "P $<0.05$ vs. Taxol. Scale bar=10 $\mu \mathrm{m}$. GTP, guanosine triphosphate; RhoA, Ras homology gene family, member A; MT, microtubule; $\mathrm{MF}$, actin/myosin microfilaments.

Co-localization between GTP-RhoA and MT in Taxol-treated cells was observed. GTP-RhoA was widely dispersed in control cells; following Taxol treatment, a GTP-RhoA bundle and an MT ring structure emerged. Furthermore, although the combination of Taxol and col partially destroyed the MT bundle, it was accompanied by the dispersal of GTP-RhoA in the cytoplasm. RhoA is a key regulator of cytoskeletal organization that regulates cell migration and invasion (26), polarity, division and early embryo development (27). In addition, Meiri et al (28) suggested that RhoGEF GEF-H1 may be sequestered in an inactive state on polymerized MTs by the dynein motor light-chain Tctex-1. They also indicated that GEF-H1 activation in response to the G-protein-coupled receptor ligands lysophosphatidic acid or thrombin is independent of MT depolymerization. However, the present study revealed that Taxol-induced cell toxicity is dependent on MT arrangement, as the disruption in MT bundling decreased GTP-RhoA protein expression levels and relieved cell cycle arrest. Notably, the present study demonstrated that decreased expression of GTP-RhoA with a specific inhibitor or by transfection with the RhoAT19N mutant plasmid had no marked effect on Taxol-induced specific MT 
bundling; however, it did reduce the Taxol-induced increase in the proportion of cells in $\mathrm{G} 2 / \mathrm{M}$ phase. These results suggested that the expression level of GTP-RhoA had no significant effect on MT arrangement. By contrast, MT rearrangement increased GTP-RhoA protein expression levels in Taxol treated cells. In conclusion, to the best of our knowledge, the results of the present study suggested that Taxol-induced MT arrangement regulates the expression levels of GTP-RhoA protein and cell cycle arrest in RCC cells. However, alterations in GTP-RhoA expression levels did not significantly influence MT arrangement.

\section{Acknowledgements}

The present study was supported by Zhejiang Provincial Medical Science Foundation of China (grant no. 2015RCB024), the Zhejiang Provincial Natural Science Foundation of China (grant no. LY13H160038), the National Natural Science Foundation of China (grant nos. 81372209 and 81071653), and the Natural Foundation of Ningbo Science and Technology Bureau of China (grant no. 2010A610047).

\section{References}

1. Cui L, Zhou H, Zhao H, Zhou Y, Xu R, Xu X, Zheng L, Xue Z, Xia W, Zhang B, et al: MicroRNA-99a induces G1-phase cell cycle arrest and suppresses tumorigenicity in renal cell carcinoma. BMC Cancer 12: 546, 2012.

2. van Spronsen DJ, de Weijer KJ, Mulders PF and De Mulder PH: Novel treatment strategies in clear-cell metastatic renal cell carcinoma. Anticancer Drugs 16: 709-717, 2005.

3. Reeves DJ and Liu CY: Treatment of metastatic renal cell carcinoma. Cancer Chemother Pharmacol 64: 11-25, 2009.

4. Van Aelst L and D'Souza-Schorey C: Rho GTPases and signaling networks. Genes Dev 11: 2295-2322, 1997.

5. Kang HG, Jenabi JM, Zhang J, Keshelava N, Shimada H, May WA, Ng T, Reynolds CP, Triche TJ and Sorensen PH: E-cadherin cell-cell adhesion in ewing tumor cells mediates suppression of anoikis through activation of the ErbB4 tyrosine kinase. Cancer Res 67: 3094-3105, 2007.

6. Cheng HL, Su SJ, Huang LW, Hsieh BS, Hu YC, Hung TC and Chang KL: Arecoline induces HA22T/VGH hepatoma cells to undergo anoikis - involvement of STAT3 and RhoA activation. Mol Cancer 9: 126, 2010.

7. Vega FM and Ridley AJ: Rho GTPases in cancer cell biology. FEBS Lett 582: 2093-2101, 2008.

8. Etienne-Manneville $\mathrm{S}$ and Hall A: Rho GTPases in cell biology. Nature 420: 629-635, 2002

9. Kamai T, Tsujii T, Arai K, Takagi K, Asami H, Ito Y and Oshima H: Significant association of Rho/ROCK pathway with invasion and metastasis of bladder cancer. Clin Cancer Res 9: 2632-2641, 2003.

10. Wang P, Xu S, Zhao K, Xiao B and Guo J: Increase in cytosolic calcium maintains plasma membrane integrity through the formation of microtubule ring structure in apoptotic cervical cancer cells induced by trichosanthin. Cell Biol Int 33: 1149-1154, 2009.

11. Jiang Q, Bai T, Shen S, Li L, Ding H and Wang P: Increase of cytosolic calcium induced by trichosanthin suppresses cAMP/PKC levels through the inhibition of adenylyl cyclase activity in HeLa cells. Mol Biol Rep 38: 2863-2868, 2011.
12. Wang P and Li JC: Trichosanthin-induced specific changes of cytoskeleton configuration were associated with the decreased expression level of actin and tubulin genes in apoptotic Hela cells. Life Sci 81: 1130-1140, 2007.

13. Gerdes JM and Katsanis N: Small molecule intervention in microtubule-associated human disease. Hum Mol Genet 14: R291-R300, 2005. [CrossRef]

14. Guo Z, Jin X and Jia H: Inhibition of ADAM-17 more effectively down-regulates the Notch pathway than that of $\gamma$-secretase in renal carcinoma. J Exp Clin Cancer Res 32: 26, 2013.

15. Wang P, Huang S, Wang F, Ren Y, Hehir M, Wang X and Cai J: Cyclic AMP-response element regulated cell cycle arrests in cancer cells. PLoS One 8: e65661, 2013.

16. Zhao K, Wang W, Guan C, Cai J and Wang P: Inhibition of gap junction channel attenuates the migration of breast cancer cells. Mol Biol Rep 39: 2607-2613, 2012.

17. He M, Cheng Y, Li W, Liu Q, Liu J, Huang J and Fu X: Vascular endothelial growth factor $\mathrm{C}$ promotes cervical cancer metastasis via up-regulation and activation of RhoA/ROCK-2/moesin cascade. BMC Cancer 10: 170, 2010.

18. Peng B, Chang Q, Wang L, Hu Q, Wang Y, Tang J and Liu X: Suppression of human ovarian SKOV-3 cancer cell growth by Duchesnea phenolic fraction is associated with cell cycle arrest and apoptosis. Gynecol Oncol 108: 173-181, 2008.

19. Yang H, Zhou J, Mi J, Ma K, Fan Y, Ning J, Wang C, Wei X, Zhao $\mathrm{H}$ and Li E: HOXD10 acts as a tumor-suppressive factor via inhibition of the RHOC/AKT/MAPK pathway in human cholangiocellular carcinoma. Oncol Rep 34: 1681-1691, 2015.

20. Bober BG and Shah SB: Paclitaxel alters sensory nerve biomechanical properties. J Biomech 48: 3559-3567, 2015.

21. Luo Y, Wang X, Wang H, Xu Y, Wen Q, Fan S, Zhao R, Jiang S, Yang J, Liu Y, et al: High bak expression is associated with a favorable prognosis in breast cancer and sensitizes breast cancer cells to paclitaxel. PLoS One 10: e0138955, 2015.

22. Khanna C, Rosenberg M and Vail DM: A review of paclitaxel and novel formulations including those suitable for use in dogs. J Vet Intern Med 29: 1006-1012, 2015.

23. Varma H, Yamamoto A, Sarantos MR, Hughes RE and Stockwell BR: Mutant huntingtin alters cell fate in response to microtubule depolymerization via the GEF-H1-RhoA-ERK pathway. J Biol Chem 285: 37445-37457, 2010.

24. Takesono A, Heasman SJ, Wojciak-Stothard B, Garg R and Ridley AJ: Microtubules regulate migratory polarity through Rho/ROCK signaling in T cells. PLoS One 5: e8774, 2010.

25. Chang YC, Nalbant P, Birkenfeld J, Chang ZF and Bokoch GM: GEF-H1 couples nocodazole-induced microtubule disassembly to cell contractility via RhoA. Mol Biol Cell 19: 2147-2153, 2008.

26. Tang Z, Zhang N, Di W and Li W: Inhibition of microtubule-associated protein 1 light chain $3 \mathrm{~B}$ via small-interfering RNA or 3-methyladenine impairs hypoxia-induced HO8910PM and HO8910 epithelial ovarian cancer cell migration and invasion and is associated with RhoA and alterations of the actin cytoskeleton. Oncol Rep 33: 1411-1417, 2015.

27. Zhang Y, Duan X, Cao R, Liu HL, Cui XS, Kim NH, Rui R and Sun SC: Small GTPase RhoA regulates cytoskeleton dynamics during porcine oocyte maturation and early embryo development. Cell Cycle 13: 3390-3403, 2014.

28. Meiri D, Marshall CB, Mokady D, LaRose J, Mullin M, Gingras AC, Ikura M and Rottapel R: Mechanistic insight into GPCR-mediated activation of the microtubule-associated RhoA exchange factor GEF-H1. Nat Commun 5: 4857, 2014. 